\title{
Metformin attenuates palmitic acid-induced insulin resistance in L6 cells through the AMP-activated protein kinase/sterol regulatory element-binding protein-1c pathway
}

\author{
WENJUN WU ${ }^{1,4^{*}}$, SUNYINYAN TANG ${ }^{2 *}$, JUNFENG SHI $^{3 *}$, WENWEN YIN $^{1}$, \\ SHU CAO ${ }^{1}$, RUIFANG BU ${ }^{4}$, DALONG ZHU ${ }^{1,2}$ and $\mathrm{YAN} \mathrm{BI}^{1,2}$ \\ ${ }^{1}$ Department of Endocrinology, Drum Tower Clinical Medical College of Nanjing Medical University; \\ ${ }^{2}$ Department of Endocrinology, Drum Tower Hospital Affiliated to Nanjing University Medical School; \\ ${ }^{3}$ Department of Oncology, Nanjing First Hospital of Nanjing Medical University, Nanjing, Jiangsu; \\ ${ }^{4}$ Department of Endocrinology, Wuxi People's Hospital of Nanjing Medical University, Wuxi, Jiangsu, P.R. China
}

Received October 18, 2014; Accepted April 9, 2015

DOI: 10.3892/ijmm.2015.2187

\begin{abstract}
AMP-activated protein kinase (AMPK) is an important effector of metformin action on glucose uptake in skeletal muscle cells. We recently reported that metformin improved insulin receptor substrate-1 (IRS-1)-associated insulin signaling by downregulating sterol regulatory element-binding protein-1c (SREBP-1c) expression. In this study, we investigated whether AMPK activation and SREBP-1c inhibition contribute to the beneficial effects of metformin on IRS-1-associated insulin signaling in L6 myotubes. L6 muscle cells were incubated with palmitic acid (PA) to induce insulin resistance and then treated with metformin and/or the AMPK inhibitor, compound C. AMPK, SREBP-1c,IRS-1 and Akt protein expression levels were determined by western blot analysis. The effects of metformin on SREBP-1c gene transcription were determined by a luciferase reporter assay. Glucose uptake was evaluated using the 2-NBDG method. In the PA-treated L6 cells, metformin treatment enhanced AMPK phosphorylation, reduced SREBP-1c expression and increased IRS-1 and Akt protein expression, whereas treatment with compound $\mathrm{C}$ blocked the effects of
\end{abstract}

Correspondence to: Professor Yan Bi or Professor Dalong Zhu, Department of Endocrinology, Drum Tower Clinical Medical College of Nanjing Medical University, 321 Zhongshan Road, Nanjing, Jiangsu 210008, P.R. China

E-mail: biyan@nju.edu.cn

E-mail: zhudalong@nju.edu.cn

*Contributed equally

Abbreviations: 2-NBDG,2-[N-(7-nitrobenz-2-oxa-1,3-diazol-4-yl)amino]-2-deoxy-D-glucose; AMPK, AMP-activated protein kinase; PA, palmitic acid; SRE, sterol regulatory element; SREBP-1c, sterol regulatory element binding protein-1c; IRS-1, insulin receptor substrate-1; GLUT4, glucose transporter 4; FAS, fatty acid synthase; GAPDH, glyceraldehyde-3-phosphate dehydrogenase

Key words: metformin, AMP-activated protein kinase, sterol regulatory element-binding protein 1c, insulin resistance, $\mathrm{L} 6$ cells metformin on SREBP-1c expression and the IRS-1 and Akt levels. Moreover, metformin suppressed SREBP-1c promoter activity and promoted glucose uptake through AMPK. The results from this study demonstrate that metformin ameliorates PA-induced insulin resistance through the activation of AMPK and the suppression of SREBP-1c in skeletal muscle cells.

\section{Introduction}

Skeletal muscle insulin resistance plays a major role in the development of postprandial hyperglycemia (1). It is well established that excess lipid accumulation in muscle leads to insulin resistance by impairing insulin signaling $(2,3)$. As a key transcription factor regulating de novo lipogenesis, the increased expression of sterol regulatory element-binding protein-1c (SREBP-1c) can result in muscular insulin resistance by promoting lipid accumulation (4-6). Nonetheless, in a recent study of ours, we reported a new mechanism through which SREBP-1c directly binds to the promoter region of insulin receptor substrate-1 (IRS-1), suppressing IRS-1 expression and, subsequently, the activation of the insulin signaling pathway (7). AMP-activated protein kinase (AMPK), a conserved serine/threonine protein kinase, is composed of a catalytic subunit $\alpha$ and two regulatory subunits $\beta$ and $\gamma$. The $\alpha$ subunit contains a threonine residue (Thr172) that is necessary for AMPK activation. AMPK is an intracellular energy sensor that plays a key role in regulating cellular metabolism (8). In muscle, AMPK has been shown to regulate insulin signaling and promote the translocation of glucose transporter 4 (GLUT4), thereby stimulating glucose uptake (9). Multiple evidence has indicated that the first-line antihyperglycemic drug, metformin, increases glucose uptake in skeletal muscle mainly through AMPK activation (10-12).

It is known that SREBP-1c is negatively regulated by AMPK. Previous studies have indicated that AMPK suppresses SREBP-1c transcription $(13,14)$ and inhibits SREBP-1c cleavage and nuclear translocation by phosphorylating SREBP-1c (15) in hepatoma cell lines and a fatty liver model, respectively. Studies have demonstrated that metformin treatment reduces hepatic lipogenesis through the the AMPK/SREBP-1c pathway $(15,16)$. 
Furthermore, our recent study indicated that metformin ameliorated IRS-1-associated insulin signaling through its inhibitory effect on SREBP-1c in skeletal muscle cells (7). Nonetheless, whether AMPK is the molecular link through which metformin acts on SREBP-1c and the subsequent insulin signaling requires clarification.

Therefore, the present study was designed to investigate the involvement of the AMPK/SREBP-1c pathway in the beneficial effects of metformin on the insulin signaling pathway in skeletal muscle cells. We found that metformin inhibited SREBP-1c expression, which was coincident with AMPK activation in PA-treated L6 myotubes. Further experiments using the AMPK inhibitor, compound C, demonstrated that AMPK activation was required for the inhibitory effects of metformin on SREBP-1c and subsequent insulin signaling. These findings provide a more precise mechanism of metformin regulation of insulin signaling in skeletal muscle cells.

\section{Materials and methods}

Materials. L6 muscle cells were obtained from the Chinese Academy of Sciences. All cell culture media and sera were purchased from Invitrogen (Carlsbad, CA, USA). Metformin and palmitic acid (PA) were obtained from Sigma-Aldrich (St. Louis, MO, USA). Compound C was obtained from EMD Millipore (Billerica, MA, USA). 2-[N-(7-nitrobenz-2oxa-1,3-diazol-4-yl)-amino]-2-deoxy-D-glucose (2-NBDG) was purchased from Cayman Chemical (Ann Arbor, MI, USA). The anti-SREBP-1c antibody (sc-8984) was obtained from Santa Cruz Biotechnology (Dallas, TX, USA). Antibodies against IRS-1 (3407), phosphorylated-IRS-1 (p-IRS-1; Tyr608/612; 09-432) and glyceraldehyde-3-phosphate dehydrogenase (GAPDH; MAB374) were obtained from EMD Millipore. Antibodies against AMPK $\alpha$ (2603), phosphorylated AMPK $\alpha$ (p-AMPK $\alpha$; Thr172; 2535), Akt (9272) and phosphorylated Akt (p-Akt; Ser473; 4060) were from Cell Signaling Technology (Danvers, MA, USA). The anti-fatty acid synthase (FAS; 610962) antibody was obtained from BD Biosciences (Franklin Lakes, NJ, USA).

Cell culture. The L6 muscle cells were cultured in DMEM supplemented with $10 \% \mathrm{FBS}$ (vol/vol) and differentiated into myotubes in differentiation medium within 6 days, as previously described (17). The monolayer of myotubes was then serum-starved in DMEM for $8 \mathrm{~h}$ and used in the following experiments. To examine the time-course effects of PA, the L6 myotubes were incubated with $0.5 \mathrm{mM}$ PA for 3, 6, 12 , 18 and $24 \mathrm{~h}$. To determine the effects of metformin and the involvement of AMPK, the L6 myotubes were treated with $0.5 \mathrm{mM} \mathrm{PA}$ in the presence or absence of metformin (1 or $10 \mathrm{mM}$ ) for $24 \mathrm{~h}$ or pre-treated with the specific AMPK inhibitor, compound $\mathrm{C}(10 \mu \mathrm{M})$, for $30 \mathrm{~min}$ and then exposed to $10 \mathrm{mM}$ metformin for $24 \mathrm{~h}$. The cells were then harvested, and the protein was extracted for western blot analysis.

Glucose uptake assay. The L6 cells were treated with the indicated compounds for $24 \mathrm{~h}$ and then incubated in low-glucose and serum-free DMEM containing $100 \mu \mathrm{M} 2-\mathrm{NBDG}$ for $1 \mathrm{~h}$ at $37^{\circ} \mathrm{C}$ in the dark. After a 10-min treatment with $100 \mathrm{nM}$ insulin, the cells were collected and the fluorescence intensity was measured at an excitation of $485 \mathrm{~nm}$ and an emission of $520 \mathrm{~nm}$ using a FACSCalibur flow cytometer (BD Biosciences). The intensity of the fluorescence reflected the 2-NBDG uptake of the cells.

PA-induced cytotoxicity assay. Cell apoptosis was detected by Annexin V-FITC/PI staining (Invitrogen). Annexin V-FITC is a fluorescence protein that binds to phosphatidylserine of the plasma membrane during early apoptosis. Propidium iodide (PI) is a fluorescent dye that binds to nuclear DNA following the rupture of the plasma membrane. L6 myotubes untreated or treated with $0.5 \mathrm{mM}$ PA for $24 \mathrm{~h}$ were resuspended in $400 \mu \mathrm{l}$ Annexin Binding Buffer, and then incubated in the dark for $15 \mathrm{~min}$ at room temperature with $4 \mu \mathrm{l}$ of Annexin V-FITC and $4 \mu \mathrm{l}$ of PI. Annexin V-FITC and PI fluorescence were measured by flow cytometry. DNA fragmentation was examined to determine late apoptosis. Total DNA was extracted from the PA-treated L6 myotubes using a DNA purification kit and analyzed on ethidium bromide (EtBr)-stained $2 \%$ agarose gels.

Western blot analysis. The treated cells were washed twice with cold phosphate-buffered saline and lysed in NP-40 lysis buffer containing a Protease Inhibitor Cocktail (Roche, Basel, Switzerland). The protein concentration was measured using the bicinchoninic acid (BCA) method. Twenty micrograms of protein per lane were loaded and separated by SDS-PAGE, transferred onto polyvinylidene fluoride membranes, blocked with $7.5 \%$ non-fat dried milk (wt/vol) in Tris-buffered saline with $0.1 \%$ Tween-20 (TBST) and incubated with primary antibodies overnight at $4^{\circ} \mathrm{C}$. The membranes were washed with TBST and incubated with the appropriate secondary antibody (goat anti-rabbit IgG, ZB-2301, ZSGB-BIO, Beijing, China and goat anti-mouse IgG, GAM0072, MultiSciences Biotech, Suzhou, China) at room temperature. The protein bands were then visualized with enhanced chemiluminescence (EMD Millipore) and quantified by densitometry (Quantity One software; Bio-Rad Laboratories, Hercules, CA, USA).

Luciferase reporter assays. The reporter plasmid, pGL3SREBP-1c, was constructed to contain the rat SREBP-1c promoter region from $-1,000$ to $+100 \mathrm{bp}$ and cloned into the KpnI/HindIII sites of the pGL3-basic luciferase vector (Promega, Madison WI, USA). The L6 cells were co-transfected with a luciferase plasmid pGL3-SREBP-1c (1,000 ng) and a pRL-TK Renilla plasmid (20 ng) using Lipofectamine 2000 (Invitrogen). At $24 \mathrm{~h}$ post-transfection, the cell medium was switched to a medium supplemented with the indicated compounds. Twelve hours later, luciferase activity was measured using the Dual-Luciferase reporter assay system (Promega). The Renilla plasmid pRL-TK was used to normalize for transfection efficiency.

Statistical analysis. All data are expressed as the means \pm SEM. Differences between groups were examined for statistical significance using the Student's t-test or one-way ANOVA. A value of $\mathrm{P}<0.05$ was considered to indicate a statistically significant difference.

\section{Results}

Metformin promotes glucose uptake through AMPK activation under PA-induced insulin-resistant states in L6 myotubes. It 
A

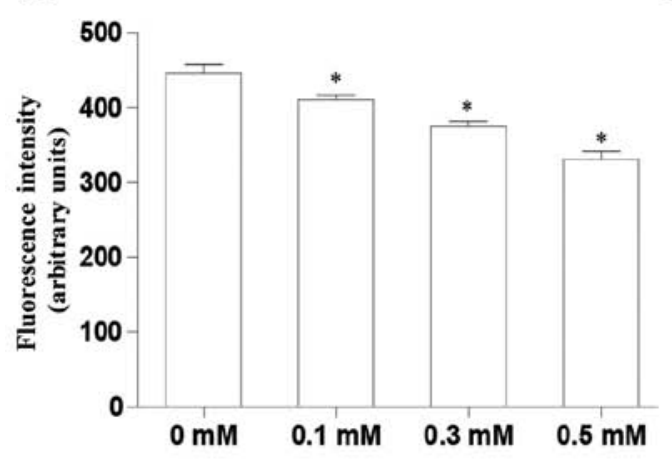

B
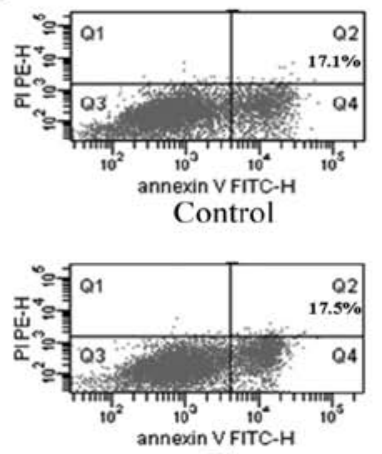

PA
C

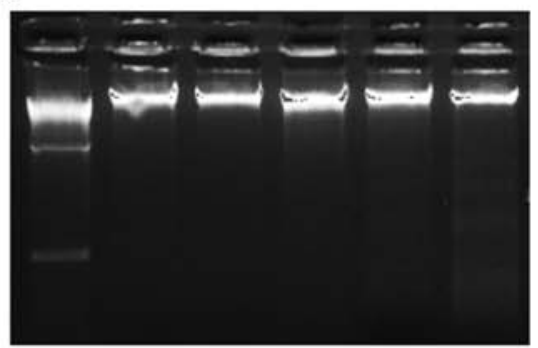

M $\quad 0 \mathrm{mM} \quad 0.1 \mathrm{mM} \quad 0.3 \mathrm{mM} 0.5 \mathrm{mM} 0.8 \mathrm{mM}$

Figure 1. Palmitic acid (PA) at the dose of $0.5-\mathrm{mM}$ led to insulin resistance in L6 myotubes without inducing cytotoxicity. (A) L6 myotubes were treated with PA $(0,0.1,0.3$ and $0.5 \mathrm{mM})$ for $24 \mathrm{~h}$. 2-NBDG uptake was decreased following PA treatment in a dose-dependent manner. (B) Untreated L6 myotubes and those treated with PA $(0.5 \mathrm{mM})$ for $24 \mathrm{~h}$ were stained with Annexin V-FITC and PI. Analysis of cell apoptosis was performed by flow cytometry. The numbers in the quadrants represent the percentage of cells in early apoptosis (Annexin $\mathrm{V}^{+} \mathrm{PI}$, lower right quadrant). (C) Late apoptosis was assayed using DNA extraction and gel electrophoresis. M, marker.
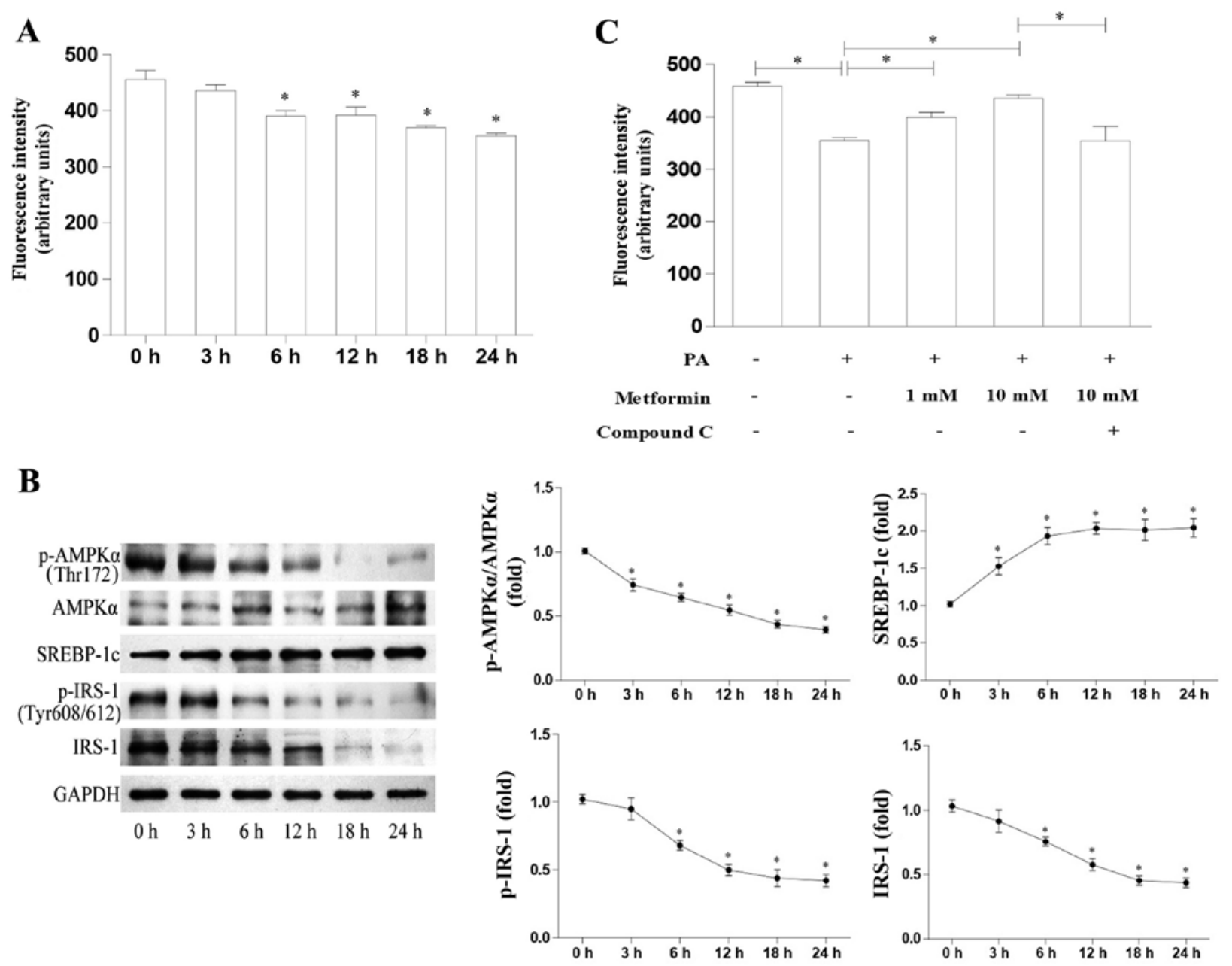

Figure 2. Metformin promotes glucose uptake through the activation of AMP-activated protein kinase (AMPK) in L6 myotubes in a palmitic acid (PA)-induced insulin-resistant state. (A) 2-NBDG uptake was decreased following PA treatment in a time-dependent manner. (B) The time-dependent effects of PA treatment on the expression of phosphorylated AMPK $\alpha$ (p-AMPK $\alpha$; Thr172), AMPK $\alpha$, sterol regulatory element-binding protein-1c (SREBP-1c), phosphorylated insulin receptor substrate-1 (p-IRS-1; Tyr608/612) and IRS-1. Protein levels (normalized to GAPDH) were measured by western blot analysis in L6 myotubes incubated with $0.5 \mathrm{mM}$ PA for $3,6,12,18$ or $24 \mathrm{~h}$. (C) Metformin enhanced 2-NBDG uptake, and this effect was attenuated by the addition of compound C. Data are presented as the means \pm SEM of at least 3 independent experiments; $\mathrm{P}<0.05$ vs. control or as indicated.

has been documented in a number of studies that PA induces insulin resistance by decreasing glucose uptake (18-20). First, in the present study, we evaluated the role of PA in glucose uptake. The results revealed that PA decreased glucose uptake in the 


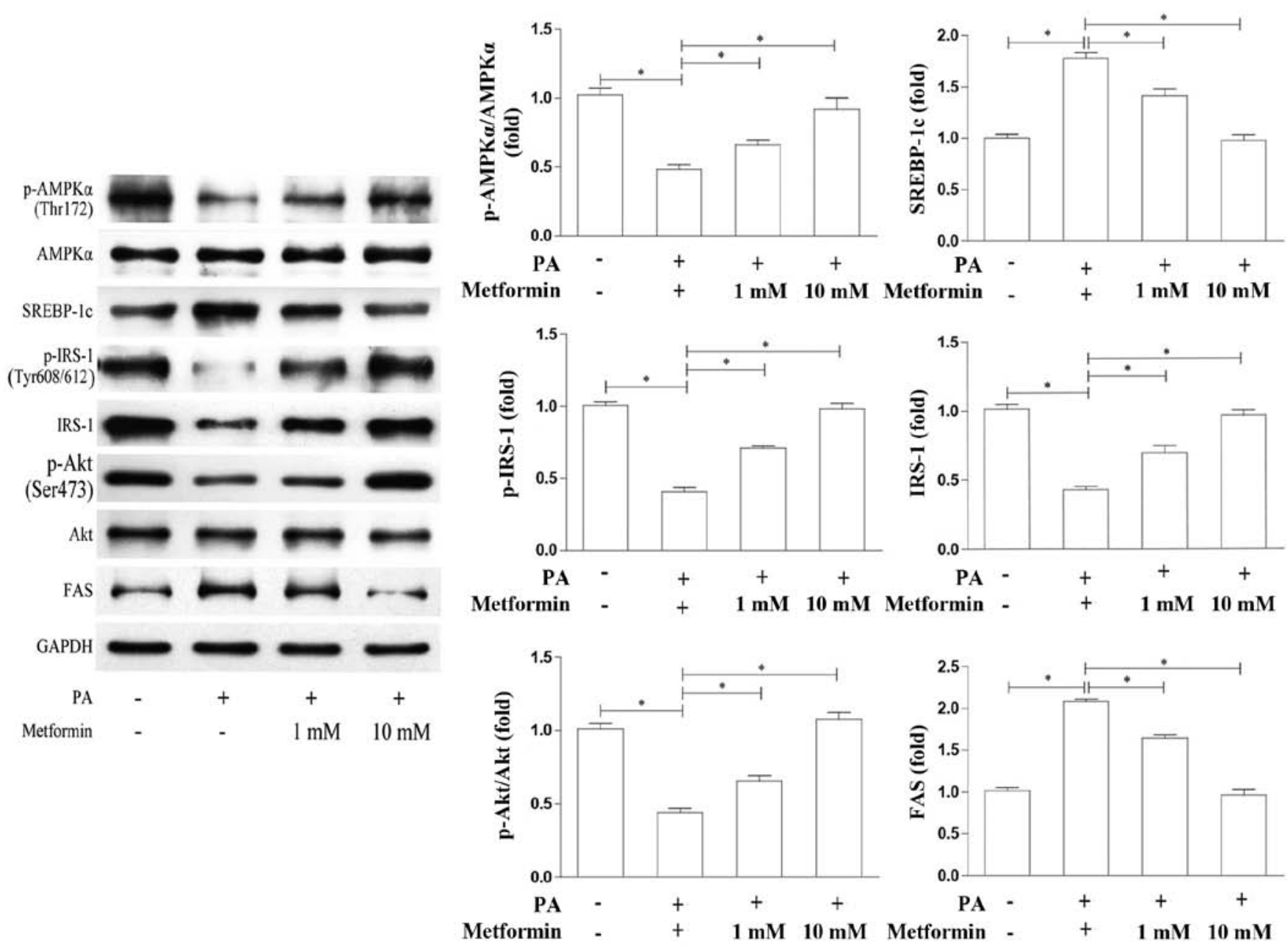

Figure 3. AMP-activated protein kinase (AMPK) is involved in the effects of metformin on the expression of sterol regulatory element-binding protein-1c (SREBP-1c) and the insulin receptor substrate-1 (IRS-1)/Akt pathway. Protein expression (normalized to GAPDH) was measured by western blot analysis in L6 myotubes treated with palmitic acid (PA) in the absence or presence of metformin $(1$ or $10 \mathrm{mM})$ for $24 \mathrm{~h}$. The graph shows the mean \pm SEM of 3 independent experiments. The significance between groups is presented as indicated; ${ }^{*} \mathrm{P}<0.05$.

L6 myotubes at doses from 0.1 to $0.5 \mathrm{mM}$ in a dose-dependent manner (Fig. 1A). Subsequently, we determined whether treatment with $0.5 \mathrm{mM}$ PA was toxic to the L6 myotubes. Annexin V-FITC/PI staining revealed that treatment with $0.5 \mathrm{mM}$ PA resulted in a similar amount of apoptosis to the control (untreated) L6 myotubes (Fig. 1B). DNA gel electrophoresis revealed that PA at doses from 0.1 to $0.5 \mathrm{mM}$ had no cytotoxic effects; however, cytotoxic effects were observed when the concentration reached $0.8 \mathrm{mM}$, which resulted in a dispersed DNA band (Fig. 1C). In the following experiments, all data were obtained using treatment with $0.5 \mathrm{mM}$ PA.

We investigated the time-course effects of PA on glucose uptake in L6 myotubes and the molecular mechanisms involved. The results revealed that PA decreased glucose uptake in a time-dependent manner (Fig. 2A). We also evaluated AMPK and SREBP-1c expression in the L6 cells treated with PA. As shown in Fig. 2B, PA decreased the p-AMPK $\alpha$ protein levels in a time-dependent manner, whereas the AMPK $\alpha$ protein levels were not affected. SREBP-1c protein expression increased significantly after $3 \mathrm{~h}$ of exposure to PA. In addition, IRS-1, as well as p-IRS-1 (Tyr608/612) expression decreased significantly after $6 \mathrm{~h}$ of PA treatment. These data suggest an inverse correlation between AMPK activation and SREBP-1c expression. The AMPK/SREBP-1c pathway may thus play a key role in PA-induced insulin resistance.

Metformin, a widely-used treatment for type 2 diabetes, has been reported to increase glucose uptake in skeletal muscle, the mechanisms of which mainly involve AMPK activation. In the present study, we investigated the role of metformin in glucose uptake and found that metformin increased glucose uptake in a dose-dependent manner compared with the PA-treated L6 cells (Fig. 2C). Furthermore, the metformin-induced enhancement of glucose uptake was attenuated following treatment with the AMPK inhibitor, compound C (Fig. 2C). Taken together, these data suggest that the metformin-induced upregulation of glucose uptake is mediated through AMPK activation.

Metformin stimulates AMPK activity, inhibits SREBP-Ic expression and activates the IRS-1/Akt pathway. In order to further investigate the specific mechanisms responsible for the metformin-induced increase in glucose uptake in skeletal muscle cells, we detected the protein levels of AMPK $\alpha$ and SREBP-1c in the PA-treated L6 cells which were treated with various concentrations of metformin. In addition, we also 


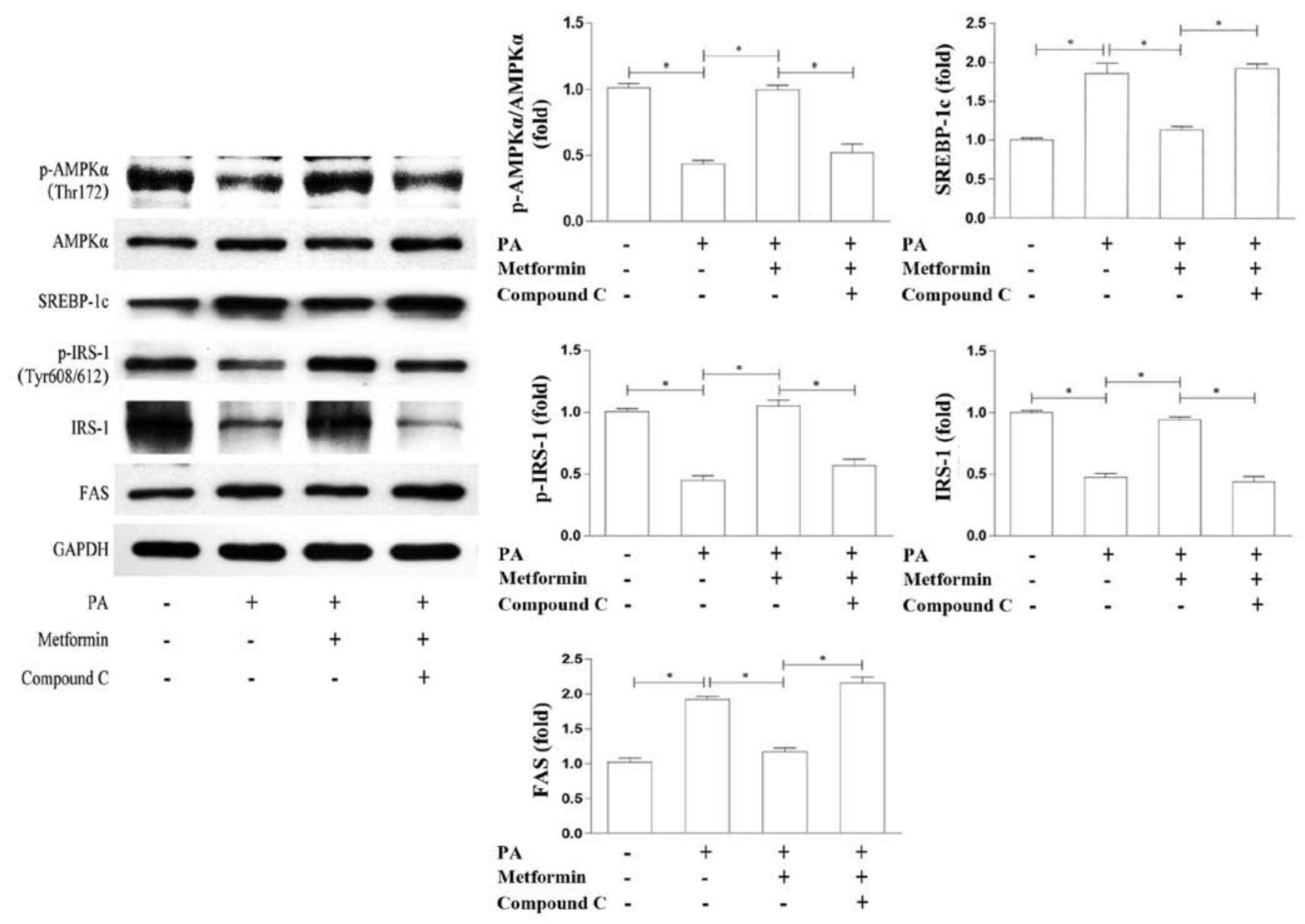

Figure 4. The AMP-activated protein kinase (AMPK) inhibitor, compound C, abolishes the effects of metformin on sterol regulatory element-binding protein-1c (SREBP-1c) and the insulin receptor substrate-1 (IRS-1)/Akt pathway. L6 myotubes were pre-treated with $10 \mu \mathrm{M}$ compound C for 30 min and then exposed to $10 \mathrm{mM}$ metformin for $24 \mathrm{~h}$. Protein was extracted and measured by western blot analysis. GAPDH was used as a loading control. The graph shows the mean \pm SEM of 3 independent experiments. The significance between groups is presented as indicated; ${ }^{*} \mathrm{P}<0.05$.

detected the expression of the SREBP-1c downstream protein, FAS, and key proteins involved in insulin signaling in muscle cells, including IRS-1 and Akt. As shown in Fig. 3, compared with the PA-treated cells not treated with metformin, the levels of AMPK $\alpha$ phosphorylation were increased in a dose-dependent manner in the cells treated with metformin, while the AMPK $\alpha$ protein levels were not affected. By contrast, the SREBP-1c and FAS protein levels were decreased in a dose-dependent manner in the cells treated with metformin. Correspondingly, the protein expression levels of IRS-1, p-IRS-1 (Tyr608/612) and p-Akt (Ser473)/Akt were increased, similar to AMPK activation. These results suggest that metformin inhibits SREBP-1c expression by activating AMPK and then the IRS-1/ Akt pathway.

The AMPK inhibitor, compound $C$, reverses the suppressive effects of metformin on SREBP-1c and impairs the activation of the IRS-1/Akt pathway. In order to examine whether the effects of metformin on SREBP-1c are AMPK-dependent, we examined the relevant proteins by co-incubation with the AMPK inhibitor, compound $\mathrm{C}$. We found that the metformininduced enhancement of AMPK $\alpha$ phosphorylation and the suppression of SREBP-1c expression were reversed by treatment with compound C (Fig. 4). In accordance with this, the protein expression of IRS-1 and p-IRS-1 (Tyr608/612) was reduced, whereas FAS expression was upregulated to levels which were similar to those observed in the PA-treated cells (Fig. 4). These results support the notion that AMPK is required in the metformin-induced suppression of SREBP-1c expression and contributes to the activation of the IRS-1/Akt pathway.

Metformin suppresses SREBP-1c promoter activity in an $A M P K$-dependent manner. In ordre to confirm that metformin regulates SREBP-1c transcription and whether its role is AMPK-dependent, we examined the effects of metformin and compound C on SREBP-1c promoter activity using a luciferase reporter assay. As shown in Fig. 5, metformin suppressed the promoter activity of SREBP-1c in a dose-dependent manner, while the AMPK inhibitor, compound C, blocked this suppression of the SREBP-1c promoter. These results demonstrate that metformin exerts an inhibitory effect on SREBP-1c promoter activity and that this is AMPK-dependent.

\section{Discussion}

Metformin has been recommended as the first-line oral agent for the treatment of type 2 diabetes (21). There are many effects 


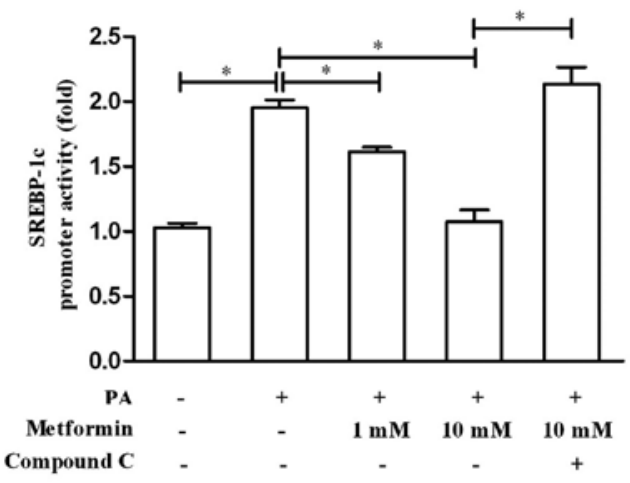

Figure 5. Metformin suppresses sterol regulatory element-binding protein-1c (SREBP-1c) promoter activity in an AMP-activated protein kinase (AMPK) dependent manner. L6 cells were co-transfected with pGL3-SREBP-1c $(1,000 \mathrm{ng})$ and pRL-TK $(20 \mathrm{ng})$ for $24 \mathrm{~h}$ and then co-incubated with palmitic acid (PA), metformin or compound $\mathrm{C}$ for a further $12 \mathrm{~h}$. The cell lysates were measured with a luciferase reporter assay. The SREBP-1c promoter activity was normalized to the Renilla luciferase activity and is expressed as the mean \pm SEM of 3 independent experiments performed in triplicate. The significance between groups is presented as indicated; ${ }^{*} \mathrm{P}<0.05$.

of metformin on glucose metabolism, including a decrease in hepatic glucose output, an increase in glucose uptake in muscle and fat, an increase in glucose utilization in the gut and an enhancement in incretin signaling (22). However, the precise molecular mechanisms of action of metformin require further clarification.

Studies on the liver have indicated that AMPK-dependent and AMPK-independent mechanisms may both account for metformin action on liver cells [reviewed in (23)]. However, studies on skeletal muscle have suggested that metformin exerts its glucose-lowering efficacy primarily through the activation of AMPK $(24,25)$. In the present study, our results support the hypothesis that metformin activates AMPK and improves glucose uptake in PA-treated L6 cells and that this beneficial effect is abolished by the AMPK inhibitor, compound C.

In addition to an increase in muscle glucose uptake, a considerable amount of evidence has indicated that the activation of AMPK by metformin also results in the inhibition of lipogenesis $(26,27)$. Studies have also established that AMPK activation leads to a reduction in lipogenesis by suppressing SREBP-1c expression $(15,16,28,29)$. Recently, the expanding roles of SREBP-1c in insulin signaling have been revealed. Studies have suggested that SREBP-1c directly inhibits IRS-2 expression and the subsequent insulin signaling pathway in the liver $(30,31)$. In a previous study of ours, it was demonstrated that SREBP-1c transcriptionally suppresses IRS-1 expression, inhibits IRS-1-associated insulin signaling and thereby decreases glucose uptake in muscle (7). In the present study, we observed that the effects of metformin treatment on insulin signaling were mediated through AMPK activation and SREBP-1c inhibition when the cells were under an insulinresistant state induced by $\mathrm{PA}$. Moreover, we found that the above-mentioned effects were AMPK-dependent. These results demonstrate that metformin increases IRS-1-associated insulin signaling partly through the AMPK/SREBP-1c pathway.

We have described the specific signaling pathway of metformin action on glucose uptake at the cellular and molecular level; however, further studies using tissues from animals or patients with insulin resistance are required in the future. In the present study, the percentage of apoptotic cells was at a high level. We speculated that the cells had a low nutritional status during the process of differentiation. In line with the majority of in vitro studies, the metformin concentration used in this study was above its physiological concentration. In this regard, a possible reason for this disparity is the differential expression of organic cation transporter (OCT) between skeletal muscle and immortalized cell lines. OCT facilitates metformin uptake into cells, and is required for the efficient action of metformin (32). Although the present study had certain limitations, our data do contribute to a better understanding of metformin action.

In conclusion, our data demonstrated that metformin enhanced IRS-1-associated insulin signaling preferentially by activating AMPK and suppressing SREBP-1c activation in L6 cells with PA-induced insulin-resistance, which led to the promotion of glucose uptake. In addition, FAS as the downstream molecule of SREBP-1c was also inhibited, which reduced lipogenesis and further contributed to the improvement in muscular insulin resistance. Therefore, the mechanism of actions of metformin revealed in the present study suggest that AMPK and the SREBP-1c pathway may serve as effective targets for the treatment of insulin resistance.

\section{Acknowledgements}

We would like to thank Professor Gong Dawei from the University of Maryland for his constructive advice during the preparation of the manuscript. The present study was sponsored by grants from the National Natural Science Foundation of China Grant Award (81270906, 81370947, 81070636), the Project of National Key Clinical Division, the China Postdoctoral Science Foundation (2012M521050), the Jiangsu Postdoctoral Science Foundation, Jiangsu Province's Key Provincial Talents Program (RC2011011), Jiangsu Province's Key Discipline of Medicine (XK201105), and the Key Project of Nanjing Medical Science and Technology Development Foundation (ZKX11017).

\section{References}

1. DeFronzo RA and Tripathy D: Skeletal muscle insulin resistance is the primary defect in type 2 diabetes. Diabetes Care 32: S157-S163, 2009.

2. Bosma M, Kersten S, Hesselink MK and Schrauwen P: Re-evaluating lipotoxic triggers in skeletal muscle: relating intramyocellular lipid metabolism to insulin sensitivity. Prog Lipid Res 51: 36-49, 2012.

3. Muoio DM: Revisiting the connection between intramyocellular lipids and insulin resistance: a long and winding road. Diabetologia 55: 2551-2554, 2012.

4. Mingrone G, Rosa G, Greco AV, Manco M, Vega N, Nanni G, Castagneto $M$ and Vidal $H$ : Intramyocitic lipid accumulation and SREBP-1c expression are related to insulin resistance and cardiovascular risk in morbid obesity. Atherosclerosis 170: 155-161, 2003.

5. Shimano H: SREBP-1c and TFE3, energy transcription factors that regulate hepatic insulin signalling. J Mol Med 85: 437-444, 2007.

6. Raghow R, Yellaturu C, Deng X, Park EA and Elam MB: SREBPs: the crossroads of physiological and pathological lipid homeostasis. Trends Endocrinol Metab 19: 65-73, 2008.

7. Bi Y, Wu W, Shi J, et al: Role for sterol regulatory element binding protein-1c activation in mediating skeletal muscle insulin resistance via repression of rat insulin receptor substrate-1 transcription. Diabetologia 57: 592-602, 2014. 
8. Mantovani J and Roy R: Re-evaluating the general(ized) roles of AMPK in cellular metabolism. FEBS Lett 585: 967-972, 2011.

9. Friedrichsen M, Mortensen B, Pehmøller C, Birk JB and Wojtaszewski JF: Exercise-induced AMPK activity in skeletal muscle: role in glucose uptake and insulin sensitivity. Mol Cell Endocrinol 366: 204-214, 2013.

10. Musi N, Hirshman MF, Nygren J, et al: Metformin increases AMP-activated protein kinase activity in skeletal muscle of subjects with type 2 diabetes. Diabetes 51: 2074-2081, 2002.

11. Zhou G, Myers R, Li Y, et al: Role of AMP-activated protein kinase in mechanism of metformin action. J Clin Invest 108 1167-1174, 2001.

12. Sajan MP, Bandyopadhyay G, Miura A, et al: AICAR and metformin, but not exercise, increase muscle glucose transport through AMPK-, ERK-, and PDK1-dependent activation of atypical PKC. Am J Physiol Endocrinol Metab 298: E179-E192, 2010.

13. Yang J, Craddock L, Hong S and Liu ZM: AMP-activated protein kinase suppresses LXR-dependent sterol regulatory elementbinding protein-1c transcription in rat hepatoma McA-RH7777 cells. J Cell Biochem 106: 414-426, 2009.

14. Yap F, Craddock L and Yang J: Mechanism of AMPK suppression of LXR-dependent Srebp-1c transcription. Int J Biol Sci 7: 645-650, 2011

15. Li Y, Xu S, Mihaylova MM, et al: AMPK phosphorylates and inhibits SREBP activity to attenuate hepatic steatosis and atherosclerosis in diet-induced insulin-resistant mice. Cell Metab 13: 376-388, 2011.

16. Jung EJ, Kwon SW, Jung BH, Oh SH and Lee BH: Role of the AMPK/SREBP-1 pathway in the development of orotic acidinduced fatty liver. J Lipid Res 52: 1617-1625, 2011.

17. Rachek LI, Musiyenko SI, LeDoux SP and Wilson GL: Palmitate induced mitochondrial deoxyribonucleic acid damage and apoptosis in L6 rat skeletal muscle cells. Endocrinology 148 293-299, 2007.

18. Dimopoulos N, Watson M, Sakamoto K and Hundal HS: Differential effects of palmitate and palmitoleate on insulin action and glucose utilization in rat L6 skeletal muscle cells. Biochem J 399: 473-481, 2006.

19. Hirabara SM, Silveira LR, Abdulkader F, Carvalho CR, Procopio $\mathrm{J}$ and Curi R: Time-dependent effects of fatty acids on skeletal muscle metabolism. J Cell Physiol 210: 7-15, 2007.

20. Zhao HL, Liu LZ, Sui Y, Ho SK, Tam SK, Lai FM, Chan JC and Tong PC: Fatty acids inhibit insulin-mediated glucose transport associated with actin remodeling in rat L6 muscle cells. Acta Diabetol 47: 331-339, 2010
21. Bailey T: Options for combination therapy in type 2 diabetes: comparison of the ADA/EASD position statement and AACE/ACE algorithm. Am J Med 126: S10-S20, 2013.

22. Cho YM and Kieffer TJ: New aspects of an old drug: metformin as a glucagon-like peptide 1 (GLP-1) enhancer and sensitiser. Diabetologia 54: 219-222, 2011.

23. Rena G, Pearson ER and Sakamoto K: Molecular mechanism of action of metformin: old or new insights? Diabetologia 56: 1898-1906, 2013.

24. Lee JO, Lee SK, Jung JH, Kim JH, You GY, Kim SJ, Park SH, Uhm KO and Kim HS: Metformin induces Rab4 through AMPK and modulates GLUT4 translocation in skeletal muscle cells. J Cell Physiol 226: 974-981, 2011.

25. Lee SK, Lee JO, Kim JH, et al: Metformin sensitizes insulin signaling through AMPK-mediated PTEN down-regulation in preadipocyte 3T3-L1 cells. J Cell Biochem 112: 1259-1267, 2011.

26. Barbero-Becerra VJ,Santiago-HernandezJJ,Villegas-LopezFA, Mendez-Sanchez N, Uribe M and Chavez-Tapia NC: Mechanisms involved in the protective effects of metformin against nonalcoholic fatty liver disease. Curr Med Chem 19: 2918-2923, 2012.

27. Fullerton MD, Galic S, Marcinko K, et al: Single phosphorylation sites in Acc1 and Acc2 regulate lipid homeostasis and the insulin-sensitizing effects of metformin. Nat Med 19: 1649-1654, 2013.

28. Quan HY, Kim do Y, Kim SJ, Jo HK, Kim GW and Chung SH: Betulinic acid alleviates non-alcoholic fatty liver by inhibiting SREBP1 activity via the AMPK-mTOR-SREBP signaling pathway. Biochem Pharmacol 85: 1330-1340, 2013.

29. Lee HI, Yun KW, Seo KI, Kim MJ and Lee MK: Scopoletin prevents alcohol-induced hepatic lipid accumulation by modulating the AMPK-SREBP pathway in diet-induced obese mice. Metabolism 63: 593-601, 2014

30. Shimomura I, Matsuda M, Hammer RE, Bashmakov Y, Brown MS and Goldstein JL: Decreased IRS-2 and increased SREBP-1c lead to mixed insulin resistance and sensitivity in livers of lipodystrophic and ob/ob mice. Mol Cell 6: 77-86, 2000.

31. Ide T, Shimano H, Yahagi N, et al: SREBPs suppress IRS-2mediated insulin signalling in the liver. Nat Cell Biol 6: 351-357, 2004.

32. Chen S, Zhou J, Xi M, Jia Y, Wong Y, Zhao J, Ding L, Zhang J and Wen A: Pharmacogenetic variation and metformin response. Curr Drug Metab 14: 1070-1082, 2013. 